\title{
Prospects for a General Theory of Evolutionary Novelty
}

\author{
DOUGLAS H. ERWIN
}

\begin{abstract}
Novelty is a topic of broad interest, with two distinct approaches within evolutionary biology. The dominant approach since Darwin has been transformationist, with novelty arising through gradual changes in morphology. The Modern Synthesis emphasized the importance of ecological opportunity rather than the source of variation, and this view has many adherents today. Yet, since well before Darwin, an alternative view has held that novelties could arise by rapid changes and many not necessarily be connected to ecological opportunity. The rise of comparative evolutionary developmental biology since 1990 has led to a resurgence of these arguments. Many case studies have documented novelties and there have been rigorous efforts to define the attributes of novelty, but there have been few attempts at a more general model. In contrast, studies of technological innovation have been replete with qualitative models since the 1930s. In this article I consider several possibilities for constructing a general model of novelty and innovation: (1) A general formal theory. (2) Commonalities between different levels, such as genes and morphology, but with sufficient differences between domains that any formal theory would be level specific. (3) Commonalities across levels but for various reasons developing a formal theory even within domains is improbable. A final alternative is that novelty and innovation may be so deeply historical that any general framework is impossible. I conclude that a common conceptual framework can be developed and serve as the foundation for simulation studies, but the importance of feedbacks and potentiating factors renders a formal model implausible.
\end{abstract}

Keywords: comparative evolutionary development, ecological opportunity, innovation, novelty.

\section{INTRODUCTION}

$\mathbf{N}$ OVELTY AND INNOVATION are topics of great interest, not only to biologists, but also to anthropologists concerned with social complexity (Hochberg et al., 2017), to economists, sociologists, and others concerned with technological evolution and economic growth (Wagner et al., 2016; Godin, 2017), to psychologists interested in the origins of human creativity (Simonton, 1999), and of course to writers, artists, architects, and musicians (North, 2013). Defining novelty and constructing a broader theory are an ongoing challenge in each of these disciplines. The sources of new morphology have been a key concern of comparative evolutionary developmental biology (evo-devo). In contrast, contributors to the Modern Synthesis,

Department of Paleobiology, MRC-121, National Museum of Natural History, Washington, District of Columbia. Santa Fe Institute, Santa Fe, New Mexico. 
such as Mayr and Simpson, and their successors downplayed the importance of variation and emphasized ecological opportunity as a critical factor in successful evolutionary novelties. Simpson's idea of adaptive radiation has been the principle explanatory framework for evolutionary novelty, although it largely focuses on taxonomic diversification. The sources of cultural and technological innovation have also attracted considerable attention, frequently relying on metaphors from biology (e.g., Godin, 2017; Hochberg et al., 2017).

Although models of technological innovation have been advanced for almost a century, and there are several models of adaptive radiations (Erwin, 2015; Stroud and Losos, 2016), there have not been attempts to integrate and test disparate views of novelty and innovation held by micro- and macroevolutionists and the evo-devo community. The ideal goal would be to identify a formal (i.e., mathematical) model of novelty and innovation across biological, cultural, and technological domains, where insights into the process from one domain could inform our understanding of other domains. (Cultural evolution is one of the most intriguing cases of evolutionary innovation.) Failing this, recognizing a common conceptual framework for novelty and innovation would be a significant advance. In this article, however, I confine myself to the (slightly) more limited goal of assessing the viability of a model within biology.

Even within evolutionary biology there are critical issues that need to be resolved at the outset of any effort to build a model. The most important of these are defining novelty and innovation with sufficient precision and establishing whether any model should focus on the product (a novelty), the process of generation, or the consequences. One could argue that part of the power of theoretical population genetics has come from a certain agnosticism about the nature of a gene, but the variety of phenomena classed as evolutionary innovations seems too broad to support such an approach. In the following sections, I distinguish novelty and innovation and characterize why they are important to understanding the history of life, briefly outline different views of novelty and innovation, and discuss possible avenues to developing either a formal model or a conceptual framework.

\section{BACKGROUND}

\subsection{Modern Synthesis views of novelty and innovation}

Darwin's view of evolutionary change was incremental; from the range of variability in a species, natural selection would favor reproduction by those individuals best suited to the current biological and physical environment. Later generations of evolutionary biologists followed Darwin in arguing that discontinuities were an artifact of extinction or failure to preserve intermediate fossils. Current views have expanded this slightly, encompassing random genetic drift, but remain fundamentally incrementalist, invoking slight changes in DNA sequences, enhancer binding, physiological parameters, or external morphology as sufficient to fuel all evolutionary change. Adherents to such microevolutionary views reject any distinction of novelty as a different phenomenon from adaptive change. Moreover, mutation and other sources of genetic variation were viewed as sufficiently frequent that they did not represent a limiting factor in the supply of morphologic novelty.

Mayr followed Darwin, viewing an evolutionary novelty as any new structure that permitted a new function (Mayr, 1960, 1963). Building on earlier suggestions by Simpson (1953), Mayr linked these novelties to opening a new adaptive zone. Ecological opportunities were seen as the primary controlling factor for evolutionary novelty, with taxonomic diversification occurring in response to new opportunities provided by creation of new niches through extinction of prior occupants, invasion of a new area, or a "key innovation" allowing access to new resources (Simpson, 1953; Losos, 2010; Stroud and Losos, 2016). These conditions generated adaptive radiations, which Simpson saw as underpinning much of evolutionary history (Simpson, 1960). Numerous models of adaptive radiations have been developed (Gavrilets and Vose, 2005; Gavrilets and Losos, 2009; Rabosky and Glor, 2010). However, it is now clear that there is no necessary link between ecological opportunity and phenotypic novelty (Donoghue and Sanderson, 2015; Erwin, 2015; Jablonski, 2017).

\subsection{Evo-devo views of novelty}

Darwin's views were not universally accepted by biologists even in the 1860s. Other biologists, including German idealistic morphologists as well as English paleontologists, acknowledged the importance 
of the evident discontinuities between species and the much greater morphologic discontinuities between larger clades, such as classes within the Mollusca. They viewed discontinuities as real and informative about patterns of evolutionary change. More recently, paleontologists studying the diversity of morphologies in fossil clades (Valentine and Erwin, 1983; Erwin, 2007; Hughes et al., 2013) have come to similar conclusions. Other recent studies of evolutionary novelty have focused on the generation of morphological variation rather than ecological opportunity. Where Darwin, Mayr, and others linked form and function, many of those in the evo-devo community sheared the two issues, focusing on form, and particularly on the origin of new, homologous characters as the basis of novelty (Muller and Wagner, 1991; Moczek, 2008; Pigliucci, 2008; Peterson and Muller, 2016).

For Moczek (2008), novelty began where homology ended. The concept of homology remains highly contested in biology but there is, by definition, a sense of continuity in a homologous character, no matter how different the various states of the character. Feathers, flowers, and vertebrate limbs each represent homologous characters, despite the great morphological variety within each class. Deep homologies exist in the developmental processes of even more disparate structures, such as limbs or eyes across bilaterian animals (Carroll 2005; Shubin et al., 2009; Tweedt and Erwin, 2015).

Until relatively recently, many paleontological studies of novelty and innovation have relied upon the generation of "higher taxa" such as orders or classes as a metric for innovation (Jablonski, 2001, 2003). Although taxic diversification is intrinsically interesting, it is a limited approach in the absence of phylogeny or analysis of novel characters (as noted by Jablonski, 2017).

\subsection{Tension between alternative views}

The central disagreement between the saltational and transformationist viewpoints is over whether evolutionary discontinuities exist, and if they do, whether explaining them requires mechanisms beyond those encompassed by the Modern Synthesis. To the transformationists, the discontinuities are either an artifact or real at the morphologic level, while change at the genetic level is gradual. The evo-devo position is that morphologic discontinuities reflect the supply of variation and the structure of regulatory patterning (Amundson, 2005).

We are thus left with an interesting conundrum. Mayr, Simpson, and others held that the supply of mutations is relatively constant and thus evolutionary innovation is largely controlled by the availability of ecological opportunity, with adaptive radiations playing a central role. In contrast, the recent interest in morphological novelty has emphasized that the nature of genetic and developmental changes plays a significant or controlling role in generating novelty, while largely ignoring the factors contributing to the evolutionary success of a novelty. Neither approach offers a unified view of the processes involved in generating novelties (however we define them) and explaining their success.

\subsection{Novelty versus innovation}

The terms novelty and innovation have been used interchangeably and previous generations of evolutionary biologists equated them with the origin of higher taxa such as the Linnean taxonomic ranks of orders, classes, or phyla. More recently, novelty has been applied to new forms, and innovation to new functions (Wagner and Lynch, 2010; Wagner, 2014b). Distinguishing form and function is indeed critical [the debate over which has primacy dates back to Cuvier and Geoffroy (Appel, 1987)]. Others have disagreed over whether to emphasize the pattern or the process (Hallgrimsson et al., 2012; Peterson and Muller, 2013). For economists and historians of technology, invention is the origin of a new technological product, process, or an organizational structure that is sufficiently distinct from similar products to warrant patent protection. Thus a slight modification to the structure of a wooden barrel is not an invention, whereas few would question the discovery of nylon in 1937 as an invention. Innovation represents the successful introduction of an invention into the economy. Invention is about technology; innovation is about economic impact. This distinction is essential in understanding technological evolution because most inventions (based on studies of patent databases) never become innovations.

A similar phenomenon occurs in biological evolution. Paleontologists have documented macroevolutionary lags between the first appearance of a phenotypic novelty and when the clade diversifies or becomes ecologically significant ( Jablonski and Bottjer, 1990; Erwin, 2015; Jablonski, 2017). For example, the lag between the origin of grasses and the spread of grasslands $>20$ million years later represents such a lag (Stromberg, 2005). I have argued that we must distinguish between novelty, the origin of newly individuated characters (following 
Wagner, 2014b), from innovation representing the point at which a clade has sufficient ecological impact that removing the node representing that clade from an ecological network (including both trophic and nontrophic interactions, thus not simply a food web) causes structural changes in the network (Erwin, 2015, 2017). (These macroevolutionary lags generate the "latent innovations" of Hochberg et al., 2017).

There are several implications of these definitions: (1) Although this does not require decoupling between novelty and innovation, it allows it, consistent with evidence from the fossil record. (2) It permits alternative routes to innovation through microevolutionary adaptive change. (3) This allows research to isolate specific tractable questions.

The challenge for any definition of novelty or innovation is how different is enough. In philosophy, this is known as Sorites paradox: take a heap of wheat (sorites means heap in ancient Greek) and begin removing grains. A heap minus one grain is still a heap, but at some point it is no longer a pile of wheat, but at what point? This paradox applies to any vague definition, and has long plagued definitions of novelty (Hochberg et al., 2017). The discontinuities already described suggest that a basis sufficient for unambiguous definitions may exist, and building a model would certainly benefit from crisp definitions of novelty and innovation.

\subsection{Types of novelties}

One of the most significant challenges to modeling evolutionary novelty is that any novelty can be characterized by three factors, only one of which can be assessed at the time the novelty arises and thus can potentially be incorporated within a model. These factors are (1) radicality, the phenotypic distance of the new trait from the ancestral condition; (2) generativity, the consequences in terms of either taxic diversification or follow-on evolutionary change (including subsequent novelty); and (3) consequentiality, the impact of the novelty through innovation. Only the first of these can be directly assessed, whereas the other two features are historically contingent consequences of a novelty. The radicality of a novelty was often the basis for assigning the rank of a new higher Linnean taxon (those involved in new classes are more radical than for a new family). Both generativity and consequentiality are closely related to Mayr and Simpson's argument that novelties allowed access to a new adaptive zone (Simpson, 1953), but because these are post hoc definitions based on the behavior of clades, rather than on the nature of the novelty itself, they are not very useful for characterizing novelty (as defined in this article).

In defining novelty as newly individuated, homologous characters Wagner distinguished two classes (Wagner, 2014b). The first, type 1, involves a new character identity, such as the insect wing. A type 2 novelty is a novel variational modality, representing deep differences between the same character in different clades, such as a butterfly wing versus the elytra of a beetle, or and feathers from epidermal scales. A related classification distinguishes type 1 novelties, a new body plan or architecture, equivalent to that which distinguishes Linnean phyla or class (this would generally involve multiple newly individuated characters); type 2 novelties are new features with no homologous character in ancestral forms (similar to Wagner's type 1 novelties), whereas type 3 is an incrementally individuated feature (Peterson and Muller, 2013, 2016).

In many cases, multiple novelties (of type 2) are required for a successful innovation, which Donoghue and Sanderson described as a "key synnovation" (Donoghue and Sanderson, 2015). Definitional purity has clearly been the agenda of some advocating restrictive definitions of novelty, whereas other have suggested that a broader view of novelty may more accurately capture evolutionary reality (Brigandt and Love, 2012). Although the articles discussed thus far focus on morphologic novelty, genomic data have exposed a variety of genomic novelties. For example, among Metazoa, four classes of genomic novelty have been recognized (Simakov and Kawashima, 2017), distinguished by the extent of sequence similarity and gene architecture (including domain order). Many novelties, at a variety of levels, occur through combinations of previously separate elements, ranging from the origin of the eukaryotic cell to co-option of regulatory subcircuits and fusion of different genes (see also Oakley, 2017).

Novelties can arise at many different levels, as new genes or gene architectures, as cell types, aspects of metabolism or physiology, at the level of regulation and development, or in the phenotype (physiology, morphology, and behavior). Although most of the discussion of novelties has focused on morphology or phenotype, novelty at one level (the phenotype) does not necessarily require, or generate, novelty at another level. A modeling project could either address the levels of novelty independently or, in a more ambitious effort, explore relationships between levels. 


\section{MODELS OF EVOLUTIONARY NOVELTY AND INNOVATION}

\subsection{Insights from models of technological innovation}

Economists and historians of technology have long been interested in technological evolution, and there is a long history of model building (often as conceptual frameworks) (Godin, 2017). In fact, the distinction between invention and innovation is credited to the economist Schumpeter (1935), which I applied to biological evolution (Erwin and Krakauer, 2004; Erwin, 2015). There has been less interest in defining novelty or innovation, and more interest in the processes generating innovation (like Mayr and Simpson, Schumpeter dismissed invention as an uninteresting problem). The earlier models of technological evolution were linear, from scientific discovery to invention to innovation, followed by diffusion through the economy, recognizing that a majority of inventions had no impact. In response to this, demand-driven models placed consumer demand as the motivating force. Linear models were quickly abandoned, however, with the recognition that feedbacks and the state of the economic system were critical variables that had not been considered (Kline, 1985; Godin, 2017). Both factors, feedback and potentiating circumstances, seem likely to be as relevant in the biological domain; neither is explicitly a part of an adaptive radiation model.

Most models of cultural change have been derived from population genetic models of biological evolution, are incremental, and do not allow discontinuities. A significant recent alternative approach comes from an exploration of the acquisition of new tools in cultural evolution. Kolodny et al. (2015) simulated four processes: (1) a "lucky-leap" discovery of a new tool; (2) expansion of the toolkit through new tools enabled by the "lucky-leap"; (3) toolkits generated by analogy to existing tools, for example, realizing that a wheel could also be used to make a potter's wheel; and (4) novel combinations of existing tools. This scheme recognized the different types of novelties that can arise in a cultural context, and in the simulations each contributes different dynamics. Although the cognitive insight needed for innovation from analogy is not useful in a broader biological context, the other three processes are relevant. Kolodny et al. ran simulations with just these three parameters, and although the results did depend upon the rates selected, they generally show a punctuated increase in the number of tools, with a gradual accumulation of new tools between punctuations (caused by the lucky-leaps) through the other two processes.

\subsection{Components of a model of novelty and innovation}

In 2015, I sketched a model of novelty and innovation involving four elements: potentiation, novelty, adaptive refinement, and innovation (Erwin, 2015, see also Erwin, 2017; Wood and Erwin, 2018). This is explicitly not a linear model, as it recognizes feedbacks and delays and the possibility of potentiation before both novelty and innovation. Potentiation before a possible innovation may not occur, or may be delayed, injecting an element of historical contingency into the process. This model is indebted to that developed for the appearance of the Cit+ mutant in Lenski's long-term Escherichia coli experimental evolution system.

3.2.1. Potentiation. In contrast to the ideas of a few decades ago, many studies have shown that the order in which mutations occur can have a substantial impact on the course of evolution. In reconstructing the appearance of a mutant form that could metabolize citrate in the medium, Lenski's group identified the Cit+ mutant, but also established that it required an earlier potentiating mutation (Blount et al., 2008; Blount et al., 2012). The potentiating mutation had also arisen in two other clonal lines, but without any $\mathrm{Cit}+$ mutant ever appearing. More generally, potentiating events include changes that are directly necessary for the generation of the novelty. General changes that are not directly associated with generating the opportunity for a novelty do not represent potentiating events. To take an extreme example, the origin of eukaryotes was a necessary precondition for the novelties leading to early animals, but it was not directly necessary (in other words, the origin of animals was not a direct consequence of the formation of eukaryotes). The origin of distal enhancers in unicellular direct ancestors of the earliest animals has a far better claim on being a potentiating change for the developmental patterning required by animals (SebePedros et al., 2016). Such prior mutations are the most obvious type of potentiation, but potentiating factors could also include developmental tools and environmental changes.

3.2.2. Novelty. Based on the discussion of types of novelties in Section 2.5, I recognize three different types of novelties. The first two correspond to Wagner's types 1 and 2 novelties, newly individuated characters and deep modifications of these characters. The third is combinations of pre-existing characters. 
There is obviously a relationship between these types, as Wagner type 2 novelties are hierarchically nested within type 1 novelties (Wagner, 2014b). The third type of novelty relies upon the existence of previous novelties or other characters. Thus the probability of the latter two types of novelties should increase through the course of a model simulation.

3.2.3. Adaptive refinement. The caricature of a "hopeful monster" approach to novelty is that a novel phenotype will be immediately viable. In reality, novelties are likely to require further adaptive refinement to become successfully incorporated into the development and ecology of an organism. Novelties may also be a trigger to further novelties, or extensive adaptive change, depending on the circumstances.

Beyond refinement of the initial novelty, and possible subsequent novelties, there is the possibility of additional potentiating events before the clade containing the novelty becomes ecologically successful. This may well be the trickiest part of this conceptual framework. Viability and continuity are critical throughout the processes of novelty and innovation (part of the reason that "hopeful monster" scenarios never achieved any traction). This new lineage must be sufficiently successful to persist. In some cases, however, the lineage may not achieve much ecological traction and thus innovation may be delayed. In contrast to potentiation associated with novelties, potentiation before an innovation is more likely to involve environmental or ecological changes that generate a setting favorable to the ecological success of the novelty.

3.2.4. Innovation. By defining innovation as ecological impact as measured by change in the structure and functioning of the ecological network (by which I mean both trophic and nontrophic interactions), this scheme focuses on the impact of a novelty within an ecosystem, rather than simply the presence of a new clade. Specifically, an innovation has occurred once the removal of the taxon from the network causes significant change to the structure and functioning of the network. For decades, paleontologists focused on the origins of higher taxa, in part, because that was what we could study because gathering reliable abundance data to assess impact is challenging (Jackson and Erwin, 2006). Although the origin of taxa can be illuminating, it is a different issue than impact. Macroevolutionary lags show the importance of focusing on innovations, and the factors responsible for them. In fact, assessing when an innovation has occurred through analysis of network dynamics will be difficult because gathering sufficient data is a challenge, and in most cases, this can be assessed more practically by noting increases in abundance (taking into account changes in preservation).

3.2.5. Consolidation. Once a novelty or novelties have been involved in a successful innovation, there will generally be additional phase in which the clade expands into other regions and into other ecological assemblages. This has been termed diffusion in the literature on technological innovation and has inspired a vast literature (Rogers, 1995). But in biology, this is within the realm of the usual ecological and evolutionary processes and requires no particular investment either conceptually or in building a model.

\subsection{Range of possible models}

There are a variety of different possible approaches to generating a common framework for novelty and innovation through the history of life. Those evolutionary biologists who reject any distinction between novelty and adaptive microevolutionary change will be satisfied with models of adaptive radiations, or with the microevolutionary combinatorics of Wagner (2011), for a critique of this approach see Erwin (2017). Alternatively, evolutionary novelties and innovations may be sufficiently different that a historical treatment is the only reasonable approach. But there are sufficient commonalities across different events that some efforts toward building a common framework may be illuminating (Wagner, 2014a; Donoghue and Sanderson, 2015; Erwin, 2017; Hochberg et al., 2017; Jablonski, 2017).

Such a common framework could take several paths: First is a general formal theory with a structure similar to quantitative population genetics. Strogatz and colleagues have recently developed such a model, but it relies upon a very different notion of novelty and innovation, one that is sufficiently general that it does not capture the issues of new homologous characters that have been so critical in biological discussions of novelty (Monechi et al., 2017). Moreover, I am concerned that this approach would not handle the historical (time-dependent) aspects of this conceptual model. Second is a simulation approach, similar 
to that of Kolodny et al. (2015). These approaches would allow exploration of different parameter values and of the relationship between different components of the model. Third, abandoning the attempt to devise a common model of novelty across levels for a model independent of the level at which the novelty develops. This would be an easier approach than the previous two paths, but would not allow exploration of the relationship between novelties at different levels. Finally, we may not yet be in a position to explore these approaches, and may need further work on a largely conceptual qualitative model that applies across novelties arising at multiple different levels, and to innovation.

\section{DISCUSSION}

Although building a model (or models) of novelty and innovation is challenging, I have argued that there is a basis for simulation models using three related types of novelties, and distinguishing between potentiation, novelty, adaptive refinement, and innovation. Several important issues remain unresolved in this framework, however. The most significant of these is working out how to express the relationship between novelties at different levels (new types of developmental regulation not necessarily expressed as morphologic novelties, for example). This will likely require hierarchical structuring of the novelties, with some probability of novelties at one level influencing another level. It will be interesting to see simulation studies that address the issue in more detail. Second, I have proposed a fairly restrictive definition of novelty. Among other consequences, this excludes changes in size as a form of novelty. One could argue that size is a property of a clade, rather than a character, and thus is better considered under innovation, but I am not sure this is entirely convincing. Decreases in the size of maniraptors were critical to the origin of birds (Lee et al., 2014) as was increased size for whales as both allowed access to new "adaptive zones." Furthermore, some have distinguished between the origins of new forms and of new functions, with those interested in the role of phenotypic and behavioral plasticity giving primacy to new functions (WestEberhard, 2003; Moczek et al., 2011).

An alternative approach to modeling, not discussed in this article, is topological, extending previous discussions of adaptive spaces. The idea of an adaptive space was central to Simpson's conception of evolutionary novelty (Simpson, 1953) and subsequent work has greatly expanded our understanding (Serrelli, 2015). But there is a significant and I think largely unresolved issue about the topology of novelty and innovation. A powerful series of articles used tRNA to explore mapping between genotype and phenotype (in this case the folded tRNA) (Stadler et al., 2001; Fontana, 2006), which have been extended to explore novelties in a variety of other networks (Wagner, 2011, 2014a).

Although this work does provide some insights into novelty, I have previously criticized it because conceptually it is dominated by a view of novelty as searching through a pre-existing space of possibilities of sequence, regulatory networks, etc. (Erwin, 2017). This ignores the generation of new evolutionary spaces as well as new means of exploring an existing space. Moreover, the phenotypic spaces are manifolds, locally Euclidean but becoming progressively less Euclidean with greater phenotypic distance. (This is evident from the fact that quantitative analyses of morphologic disparity become increasingly difficult with greater differences in form. So, hidden within the language of the "lucky-leaps" of Kolodny et al. (2015) and the type 1 novelties already mentioned is a difficult topological problem, one that is not addressed by this model and which requires considerable further work (but see Mitteroecker and Huttegger, 2009).

Even if a conceptual framework for novelty and innovation can be constructed, that is only the first step in addressing a much richer variety of questions about this aspect of the history of life. Distinguishing between novelty, innovation, diversification of taxa, the origin of higher taxa, and ecological impact is important, but it does not resolve issues of causal interactions between these variables. Empirically we distinguish novelty from innovation because of evidence they are not necessarily coupled. But among cases where they are linked, is there a common causal connection? Similarly, novelty at one level need not entail novelty at another level. There are several examples of where co-option of a gene regulatory network (GRN) to a new developmental address generates a new morphology (Erwin and Davidson, 2009; Glassford et al., 2015; Peter and Davidson, 2015); while the developmental timing of the GRN is new, the novelty lies at the level of morphology. Can we identify conditions where coupling between levels is more or less likely?

We currently understand too little of the mechanisms to know whether novelties at different levels are generated in different ways. The major motivating question behind recent studies of novelty in the evo- 
devo community involves whether novelty depends on different sorts of variation than that responsible for most adaptive evolutionary change. Finally, the entire effort to build any model of evolutionary novelty and innovation assumes that the underlying processes are time homogeneous, in the sense that the probability of novelty, and of successful innovation, has not varied over time. Although the question remains unresolved, there is evidence suggesting that their pace has changed over time (Erwin, 2011). If this is so, it will be of great interest to establish the factors responsible for such changes.

\section{ACKNOWLEDGMENTS}

This study is an outgrowth of a symposium at the Jacques Loeb Centre of the Ben-Gurion University, organized by Ute Deichmann. I appreciate comments on an earlier draft from U. Deichmann and M. Hochberg.

\section{AUTHOR DISCLOSURE STATEMENT}

The author declares there are no competing financial interests.

\section{REFERENCES}

Amundson, R. 2005. The Changing Role of the Embryo in Evolutionary Thought. Cambridge University Press, Cambridge.

Appel, T.A. 1987. The Cuvier-Geoffroy Debate. French Biology in the Decades Before Darwin. Oxford University Press, Oxford.

Blount, Z.D., Barrick, J.E., Davidson, C.J., et al. 2012. Genomic analysis of a key innovation in an experimental Escherichia coli population. Nature 489, 513-518.

Blount, Z.D., Borland, C.Z., and Lenski, R.E. 2008. Historical contingency and the evolution of a key innovation in an experimental population of Escherichia coli. Proc. Natl. Acad. Sci. U. S. A. 105, 7899-7906.

Brigandt, I., and Love, A.C. 2012. Conceptualizing evolutionary novelty: Moving beyond definitional debates. J. Exp. Zool. (Mol. Dev. Evol.) 318B, 417-427.

Carroll, S.B. 2005. Evolution at two levels: On genes and form. PLoS Biol. 3, 1159-1166.

Donoghue, M.J., and Sanderson, M.J. 2015. Confluence, synnovation, and depauperons in plant diversification. New Phytol. 207, 260-274.

Erwin, D.H. 2007. Disparity: Morphological pattern and developmental context. Palaeontology 50, 57-73.

Erwin, D.H. 2011. Evolutionary uniformitarianism. Dev. Biol. 357, 27-34.

Erwin, D.H. 2015. Novelty and innovation in the history of life. Curr. Biol. 25, R930-R940.

Erwin, D.H. 2017. The topology of evolutionary novelty and innovation in macroevolution. Phil. Trans. R. Soc. Lond B Biol. Sci. 372, 20160422.

Erwin, D.H., and Davidson, E.H. 2009. The evolution of hierarchical gene regulatory networks. Nat. Rev. Genet. 10, 141-148.

Erwin, D.H., and Krakauer, D.C. 2004. Insights into innovation. Science 304, 1117-1119.

Fontana, W. 2006. Topology of the possible, 67-84. In Wimmer, A., and Kossler, R., eds. Understanding Change: Models, Methodologies and Metaphor. Palgrave Macmillan UK, Houndmills, UK.

Gavrilets, S., and Losos, J.B. 2009. Adaptive radiation: Contrasting theory with data. Science 323, 732-737.

Gavrilets, S., and Vose, A. 2005. Dynamic patterns of adaptive radiation. Proc. Nat. Acad. Sci. U. S. A. 102, 1804018045.

Glassford, W.J., Johnson, W.C., Dall, N.R., et al. 2015. Co-option of an ancestral Hox-regulated network underlies a recently evolved morphological novelty. Dev. Cell 34, 520-531.

Godin, B. 2017. Models of Innovation: The History of an Idea. The MIT Press, Cambridge, MA.

Hallgrimsson, B., Jamniczky, H.A, Young, N.M., et al. 2012. The generation of variation and the developmental basis for evolutionary novelty. J. Exp. Zool. (Mol. Dev. Evol.) 318B, 501-517.

Hochberg, M.E., Marquet, P.A., Boyd, R., et al. 2017. Innovation: An emerging focus from cells to societies. Phil. Trans. Roy. Soc. Lond B Biol. Sci. 372, 20160414

Hughes, M., Gerber, S., and Wills, M.A. 2013. Clades reach highest morphologic disparity early in their evolution. Proc. Nat. Acad. Sci. U. S. A. 110, 13875-13879. 
Jablonski, D. 2001. Origin of evolutionary novelties, 162-166. In Briggs, D.E.G. and Crowther, P.R., eds. Palaeobiology II. Blackwell, Oxford.

Jablonski, D. 2003. Evolutionary innovations in the fossil record: Patterns in time and space. Int. Comp. Biol. $43,969$. Jablonski, D. 2017. Approaches to macroevolution: General concepts and origin of variation. Evol. Biol. 44, 427-450.

Jablonski, D., and Bottjer, D.J. 1990. The origin and diversification of major groups: Environmental patterns and macroevolutionary lags, 17-57. In Taylor, P.D., and Larwood, G.P., eds. Major Evolutionary Radiations. Clarendon Press, Oxford.

Jackson, J.B.C., and Erwin, D.H. 2006. What can we learn about ecology and evolution from the fossil record. Trends Ecol. Evol. 21, 322-336.

Kline, S.J. 1985. Innovation is not a linear process. Res. Manage. 28, 36-45.

Kolodny, O., Creanza, N., and Feldman, M.W. 2015. Evolution in leaps: The punctuated accumulation and loss of cultural innovations. Proc. Nat. Acad. Sci. U. S. A. 112, 6762-6769.

Lee, M.S., Cau, A., Naish, D., et al., 2014. Sustained miniaturization and anatomical innovation in the dinosaurian ancestors of birds. Science 345, 562-566.

Losos, J.B. 2010. Adaptive radiation, ecological opportunity, and evolutionary determinism. Am. Nat. 175, 623-639.

Mayr, E. 1960. The emergence of novelty, 349-380. In Tax, S., ed. The Evolution of Life. University of Chicago Press, Chicago.

Mayr, E. 1963. Animal Species and Evolution. Belknap Press, Cambridge, MA.

Mitteroecker, P., and Huttegger, S.M. 2009. The concept of morphospaces in evolutionary and developmental biology: Mathematics and metaphors. Biol. Theory 4, 54-67.

Moczek, A.P. 2008. On the origins of novelty in development and evolution. BioEssays 30, 432-447.

Moczek, A.P., Sultan, S., Foster, S., et al. 2011. The role of developmental plasticity in evolutionary innovation. Proc. $R$. Soc. Lond B 278, 2705-2713.

Monechi, B., Ruiz-Serrano, A., Tria, F., et al., 2017. Waves of novelties in the expansion into the adjacent possible. PLoS One 12, e0179303.

Muller, G.B., and Wagner, G.P. 1991. Novelty in evolution: Restructuring the roncept. Ann. Rev. Ecol. Syst. 22, 229256.

North, M. 2013. Novelty: A History of the New. University of Chicago Press, Chicago.

Oakley, T.H. 2017. Furcation and fusion: The phylogenetics of evolutionary novelty. Dev. Biol. 431, 69-76.

Peter, I.S., and Davidson, E.H. 2015. Genomic Control Processes: Development and Evolution. Academic Press, London.

Peterson, T., and Muller, G.B. 2013. What is evolutionary novelty? Process versus character based definitions. J. Exp. Zool. (Mol. Dev. Evol.) 320B, 345-350.

Peterson, T., and Muller, G.B. 2016. Phenotypic novelty in EvoDevo: The distinction between continuous and discontinuous variation and its importance in evolutionary theory. Evol. Biol. 43, 314-333.

Pigliucci, M. 2008. What, if anything, is an evolutionary novelty? Phil. Sci. 75, 887-898.

Rabosky, D.L., and Glor, R.E. 2010. Equilibrium speciation dynamics in a model adaptive radiation of island lizards. Proc. Nat. Acad. Sci. U. S. A. 107, 22178-22183.

Rogers, E.M. 1995. Diffusion of Innovations. Free Press, New York, NY.

Schumpeter, J.A. 1935. The analysis of economic change. Rev. Eco. Stat. 17, 2-10.

Sebe-Pedros, A., Ballare, C., Parra-Acero, H., et al. 2016. The dynamic regulatory genome of Capsaspora and the origin of animal multicellularity. Cell 165, 1224-1237.

Serrelli, E. 2015. Visualizing macroevolution: From adaptive landscapes to compositions of multiple spaces, 113-162. In Serrelli, E., and Grontier, N. eds. Macroevolution. Springer, Heidelberg.

Shubin, N., Tabin, C., and Carroll, S. 2009. Deep homology and the origins of evolutionary novelty. Nature 457, 818-823.

Simakov, O., and Kawashima, T. 2017 Independent evolution of genomic characters during major metazoan transitions. Dev. Biol. 427, 179-192.

Simonton, D.K. 1999. Origins of Genius: Darwinian Perspectives on Creativity. Oxford University Press, Oxford.

Simpson, G.G. 1953. The Major Features of Evolution. Columbia University Press, New York.

Simpson, G.G. 1960. The history of life, 117-180. In Tax, S., ed. The Evolution of Life. University of Chicago Press, Chicago.

Stadler, B.M.R., Stadler, P.F., Wagner, G.P., et al. 2001. The topology of the possible: Formal spaces underlying patterns of evolutionary change. J. Theor. Biol. 231, 2241-2274.

Stromberg, C.A.E. 2005. Decoupled taxonomic radiation and ecological expansion of open-habitat grasses in the Cenozoic of North America. Proc. Nat. Acad. Sci. U. S. A. 102, 11980-11984.

Stroud, L.T., and Losos, J.B. 2016. Ecological opportunity and adaptive radiation. Ann. Rev. Ecol. Evol. Syst. 47, 507532.

Tweedt, S.M., and Erwin, D.H. 2015. Origin of metazoan developmental toolkits and their expression in the fossil record, 47-77. In Ruiz-Trillo, I., and Nedelcu, A.M., eds. Evolution of Multicellularity. Academic Press, London. 
Valentine, J.W., and Erwin, D.H. 1983. Patterns of diversification of higher taxa: A test of macroevolutionary paradigms, 220-233. In Chaline, J., ed. Modalities et Rhythmes de L'Evolution Mechanismes de Speciatio. CNRS, Paris. Wagner, A. 2011. The Origins of Evolutionary Innovations. Oxford University Press, Oxford.

Wagner, A. 2014a. Arrival of the Fittest. Penguin, New York.

Wagner, A., Ortman, S., and Maxfield, R. 2016. From the primordial soup to self-driving cars: Standards and their role in natural and technological innovation. J. R. Soc. Interface 13. DOI:10.1098/rsif.2015.1086.

Wagner, G.P. 2014b. Homology, Genes, and Evolutionary Innovation. Princeton University Press, Princeton, NJ.

Wagner, G.P and Lynch, V.J. 2010. Evolutionary novelties. Curr. Biol. 20, R48-R52.

West-Eberhard, M.J. 2003. Developmental Plasticity and Evolution. Oxford University Press, Oxford.

Wood, R., and Erwin, D.H. 2018. Innovation not recovery: Dynamic redox promotes metazoan radiations. Biol. Rev. 93, 863-873.

Address correspondence to: Dr. Douglas H. Erwin

Department of Paleobiology, MRC-121 National Museum of Natural History

P.O. Box 37012

Washington, DC 20013-7012

and

Santa Fe Institute

1399 Hyde Park Road

Santa Fe, NM 87501

E-mail: erwind@si.edu 